\title{
Positioning technology based on the shadow of the Sun Yumeng Wang ${ }^{1, a}$ \\ ${ }^{1}$ School of North China Electric Power University(Baoding), Hebei 071000, China; aydfz2010@qq.com
}

Keywords: Sun elevation angle; the least square method; over-determined equation; fitting

\begin{abstract}
The mathematical problem of the changes of the shadow length of the straight bar according to the passage of time can be solved by transforming the problem to solve the sun elevation angle corresponding to the time. The sun elevation angle is determined by the local time and Sun's declination. By using the formula of sun elevation angle and the measuring time and the local latitude and longitude, the Shadow length variation curve can be drawn. Then, a general form of equation can be achieved by fitting the Shadow length variation curve and the coefficient of the fitting curve can be solved by using the collected measuring data. The minimum shadow length can be found and the corresponding time which means the local time at noon can be recorded. So that the longitude can be solved. The measuring data can be used to construct the over-determined equation, the local latitude can be solved by the least square method. This positioning technology can be used widely in daily life.
\end{abstract}

\section{Introduction}

With the development of image processing technology, the positioning technology based on the shadow of the Sun has raised our interests. This article introduces a method to position easily

\section{Basic assumptions}

1. The data according to the observation are reliable

2. The projection surface of the straight bar is flat.

3. Without considering the impact on the straight bar and the shadow of the straight bar caused by the external factors such as wind and other natural factors.

4. The Sun's declination is the same in one day.

\section{Terminology and Definitions}

Table1.Symbol description

\begin{tabular}{|c|c|c|}
\hline Parameter & & Meaning \\
\hline l & & The length of the straight bar \\
\hline$\varphi$ & The & Geomagnetic latitude of the observation points \\
\hline$\beta$ & & The hour angle of the sun \\
\hline$\alpha$ & & Sun elevation angle \\
\hline$\theta$ & & The day angle \\
\hline$\sigma$ & & Sun's declination \\
\hline$t_{B}$ & & The Standard Time of Beijing \\
\hline$J_{d}$ & The & Geomagnetic longitude of the observation points \\
\hline$t_{p}$ & & Civil time \\
\hline$t_{Z}$ & & Solar time \\
\hline
\end{tabular}




\section{Model Description and Method of Solution}

(1)Model of the shadow length

According to the geometric relationship, we can see that

$$
d=\frac{l}{\tan \alpha}
$$

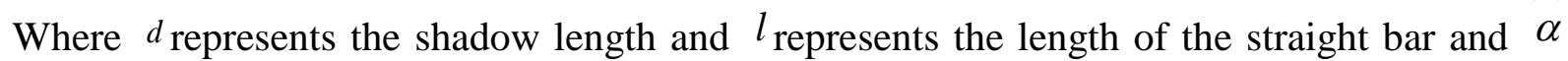
represents the sun elevation angle. Since that $l$ is a fixed value, it is important to solve the value of the sun elevation angle. The sun elevation angle can be calculated by the following formula:

$$
\sin \alpha=\sin \varphi \sin \sigma+\cos \varphi \cos \sigma \cos \beta
$$

Where $\varphi$ represents geomagnetic latitude and $\sigma$ represents Sun's declination and $\beta$ represent the hour angle of the Sun. According to the related document ${ }^{[1]}$,

$$
\begin{gathered}
\sigma=0.3723+23.2567 \sin \theta+0.1149 \sin 2 \theta-0.1712 \sin 3 \theta-0.758 \cos \theta \\
+0.3656 \cos 2 \theta+0.0201 \cos 3 \theta \\
\theta=2 \pi k / 365.2422 \\
k=N-N_{0}
\end{gathered}
$$

Where $\theta$ represents the day angle and $N$ represents the cumulative days ${ }^{[2]}$. When we calculate the the hour angle $\beta$, the following formulas can be used:

$$
\begin{gathered}
E_{t}=(0.0028-1.9857 \sin \theta+9.9059 \sin 2 \theta-7.0924 \cos \theta-0.6882 \cos 2 \theta) / 60 \\
t_{p}=t_{B}-8+J_{d} / 15 \\
t_{z}=t_{p}+E_{t} \\
\beta=\left(t_{z}-12\right) \times 15^{\circ}
\end{gathered}
$$

So that the the shadow length can be calculated by using the formula(1). we can use the MATLAB to draw the shadow length variation curve.

(2)Construct the rectangular coordinate system

We regard the center of the bottom of the straight bar and construct the rectangular coordinate system as the following figure shows:

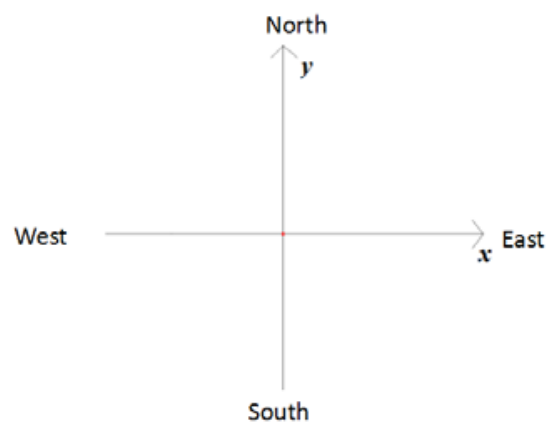

(3)Model of positioning

A general form of equation can be achieved by fitting the Shadow length variation curve and the coefficient of the fitting curve can be solved by using the collected measuring data. We select the minimum shadow length and the corresponding time and regard it as the local time at noon. Then, we substitute this time into the formula(7) and calculate the local longitude.

The local latitude can also be achieved by selecting two time and the formulas in the model of the shadow length. However, this method has a great inaccuracy because the local latitude often changes consistently at different latitude positions under the same longitude. So that the formulas of Sun elevation angle and the solar Azimuth can be simultaneously used as the following shows 


$$
\left\{\begin{array}{l}
\sin \alpha=\sin \varphi \sin \sigma+\cos \varphi \cos \sigma \cos \beta \\
\cos A=(\sin \alpha \sin \varphi-\sin \sigma) /(\cos \alpha \cos \beta) \\
\sin A=\cos \sigma \sin \beta / \cos \alpha
\end{array}\right.
$$

And we achieve the corresponding formula:

$$
-\cos \varphi \tan A \tan \sigma+\cos \beta \sin \varphi \tan A=\sin \beta
$$

Where A represents the solar Azimuth.according to the formula:

$$
A=\arctan \left(\frac{y_{s}}{x_{s}}\right)
$$

We can calculate the solar Azimuth to the corresponding time. By using the measuring data(assuming the number of the data is 21), the solar Azimuth ${ }^{[3]}$, the Sun's declination and the hour angle of the Sun can be obtained. Then, the over determined equations can be constructed by substituting these data to formula(11) as follows:

$$
\begin{gathered}
-\cos \varphi \tan A_{1} \tan \sigma+\cos \beta_{1} \sin \varphi \tan A_{1}=\sin \beta_{1} \\
-\cos \varphi \tan A_{2} \tan \sigma+\cos \beta_{2} \sin \varphi \tan A_{2}=\sin \beta_{2} \\
\cdot \\
\cdot \\
-\cos \varphi \tan A_{21} \tan \sigma+\cos \beta_{21} \sin \varphi \tan A_{21}=\sin \beta_{21}
\end{gathered}
$$

So that the local latitude can be solved by the least square method ${ }^{[4]}$. Therefore, we have access to the longitude and latitude of the observation points.

\section{The test of the model}

By using MATLAB toolbox Fourier approximation method for fitting the Shadow length variation curve, It shows that the correlation coefficient is a value close to 1 which means that the fitting effect is good.

\section{Evaluation and Extension of the model}

\section{Strengths}

(1) In order to achieve better accuracy, the over-determined equations containing 21 equations are constructed by using all the data that are offered according to the observation and measuring.

(2)The problem which exists in the rectangular space coordinate is transformed to the rectangular coordinate system, so that the calculation amounts are greatly reduced.

(3)The physical problem of solving the changes of the shadow lengths of the straight bar according to the passage of time is transformed to the problem of solving the sun elevation angle, so that the model can be widely used.

2.Weaknesses

(1)When calculating the length of the shadow, the refraction of light in the atmosphere is ignored. Thus, the result may cause errors.

(2)The Sun's declination changes slightly in a day. The result will not be accurate enough if we assume that it is all the same all along the day.

3.Extension of the model

By using simple knowledge of geography and mathematics, the information of the position can be obtained by a straight bar. Using similar methods, after understanding the geographical knowledge of other planets, this technology can be applied to other planets to position the place too. 


\section{References}

[1]Wang Bingzhong, the calculating principle of Solar zenith angle and azimuth according to date, time, latitude and longitude, and local computing.

http://blog.sina.com.cn/s/blog_4a1c6f7f0100062y.html, September 2015.

[2] The calculation used in solar irradiance categories related parameters, Ningxia Engineering Technology, Vol. 14: No. 1, March 2015.

[3] He Xiaolei, Zhuhe Jun, Li Jianying, Ding Lei, solar azimuth formula Solution and Application, Solar Technology, Vol. 29: No. 1, January 2008.

[4] Si ShouKui, Sun YuxiQing, mathematical modeling algorithms and applications, Beijing Aoxin Printing Printing: National Defense Industry Press, 2015. 